\title{
Food for thought: endocannabinoid modulation of lipogenesis
}

\author{
Aron H. Lichtman ${ }^{1}$ and Benjamin F. Cravatt ${ }^{2}$
}

\begin{abstract}
1Department of Pharmacology and Toxicology, Medical College of Virginia Campus, Virginia Commonwealth University, Richmond, Virginia, USA. ${ }^{2}$ The Skaggs Institute for Chemical Biology and Departments of Cell Biology and Chemistry, The Scripps Research Institute, La Jolla, California, USA.
\end{abstract}

\begin{abstract}
An emerging body of evidence implicates peripheral and central endocannabinoid pathways in the regulation of feeding behavior and body weight. A report in this issue of the JCI demonstrates the presence of a common endocannabinoid-regulated molecular pathway for peripheral lipogenic and central appetitive regulation (see the related article beginning on page 1298). This pathway involves the activation of the transcription factor SREBP-1c and its associated enzymes, acetyl-CoA carboxylase- 1 and fatty acid synthase, in the liver and hypothalamus. Activation of cannabinoid receptor $1\left(\mathrm{CB}_{1}\right)$ in liver plays a key role in increased serum lipid production, fatty liver, and possibly diet-induced obesity. Conversely, stimulation of these receptors in the hypothalamus may lead to an increase in food consumption. Thus, targeting both of these pathways with $\mathrm{CB}_{1}$ antagonists could promote sustained weight loss and favorable serum lipid profiles in obese patients.
\end{abstract}

Cannabis has been used since antiquity for the treatment of many ailments, including pain, rheumatoid arthritis, epilepsy, and eating disorders. William O'Shaughnessy played a leading role in introducing this substance to Western medicine in the middle of the eighteenth century. One of the many pharmacological properties of cannabis he described was the induction of a "remarkable increase of appetite" (1). Over 120 years later, Gaoni and Mechoulam identified the structure $\Delta^{9}$-tetrahydrocannabinol $\left(\Delta^{9}\right.$-THC) as the primary psychoactive constituent of cannabis (2). A considerable amount of preclinical research validated these early reports of the effects of cannabis on feeding by demonstrating that low-to-moderate doses of $\Delta^{9}$-THC and other cannabinoid receptor agonists increase feeding or foodmotivated behavior (3). Importantly, administration of oral $\Delta^{9}$-THC (Marinol) was found to have some efficacy in treating the AIDS wasting syndrome by enhancing appetite and preventing weight loss (4).

$\Delta^{9}$-THC, along with other naturally occurring and synthetic cannabinoids, binds to

Nonstandard abbreviations used: $\Delta^{9}$-THC; $\Delta^{9}$-tetrahydrocannabinol; 2-AG, 2-arachidonoylglycerol; ACC1, acetyl-CoA carboxylase- $1 ; \mathrm{CB}_{1}$, cannabinoid receptor 1 ; FAAH, fatty acid amidohydrolase; FAS, fatty acid synthase.

Conflict of interest: The authors have declared that no conflict of interest exists.

Citation for this article: J. Clin. Invest. 115:1130-1133 (2005). doi:10.1172/JCI200525076
2 separate $G$ protein-coupled receptors: cannabinoid receptor $1\left(\mathrm{CB}_{1}\right)$, which is located in both the CNS and periphery (5), and $\mathrm{CB}_{2}$, which is found primarily on cells of the immune system (6). These receptors and their endogenous ligands, which include the fatty acid amide $N$-arachidonoyl ethanolamine (anandamide) and the monoacylglycerol 2-arachidonoylglycerol (2-AG) $(7,8)$, together constitute an endogenous cannabinoid (endocannabinoid) system. This system has been proposed to modulate several physiological functions, including pain $(9,10)$, cognition $(11,12)$, drug dependence (13), excitotoxicity (14), and feeding (15).

\section{Endocannabinoid regulation of feeding and lipogenesis}

A common experimental strategy to investigate whether the endocannabinoid system tonically modulates a physiological system is to disrupt $\mathrm{CB}_{1}$ signaling using either the $\mathrm{CB}_{1}$ antagonist SR141716 (Rimonabant) (16) or $\mathrm{CB}_{1}^{-/-}$ mice $(13,17)$. SR141716 administration has been shown to have an anorexic action in a variety of rodent models of feeding (3). In rats, it caused a dose-dependent decrease in consumption of either sucrose pellets or sucrose solution, but had limited effects on the intake of standard chow or water (18), which suggests that the endocannabinoid system plays a role in the increased consumption of palatable substances. Similarly, Di Marzo and colleagues (15) reported that SR141716-treated mice and $\mathrm{CB}_{1}{ }^{-/-}$ mice ate significantly less than did control mice following 18 hours of food deprivation. They also found that $o b / o b$ and $d b / d b$ mice and Zucker rats, genetic rodent models of obesity, possessed elevated levels of endocannabinoids in the hypothalamus compared with those in nonobese control mice, while no differences were found in other brain regions (e.g., the cerebellum). The observation that SR141716 decreases food intake in obese animals suggests that endocannabinoids in the hypothalamus may play a role in hyperphagic responses. Other evidence indicates that endocannabinoids are involved in the neural circuitry of feeding regulated by leptin, a hormone that plays a key role in modulating food intake and body fat. Whereas leptin-deficient mice become obese and exhibit increased levels of endogenous cannabinoids in the hypothalamus, administration of leptin leads to a decrease in feeding and a concomitant reduction of anandamide and 2-AG expression in the hypothalamus (15). Conversely, $\mathrm{CB}_{1}^{-/-}$mice possess significantly decreased plasma levels of leptin and have reduced body fat, but exhibit enhanced sensitivity to exogenously administered leptin compared with that in wild-type mice $(19,20)$.

Cota and colleagues proposed that the endocannabinoid system plays a dual role in regulating body weight through a central orexigenic effect and in the regulation of peripheral lipogenesis (19). While stimulation of the endocannabinoid system can lead to increases in caloric consumption depending on the type of food available and whether the animal has been fasted, the number of calories consumed does not account for body weight differences found between SR141716-treated or $\mathrm{CB}_{1}{ }^{-/}$animals and controls. Instead, several lines of evidence suggest a peripheral role for endocannabinoids in the regulation of lipogenesis and body weight in adult animals. SR141716 consistently alters hormone and serum lipid levels associated with animal models of obesity $(21,22)$. In addition, the cannabinoid receptor agonist WIN-55,212-2 


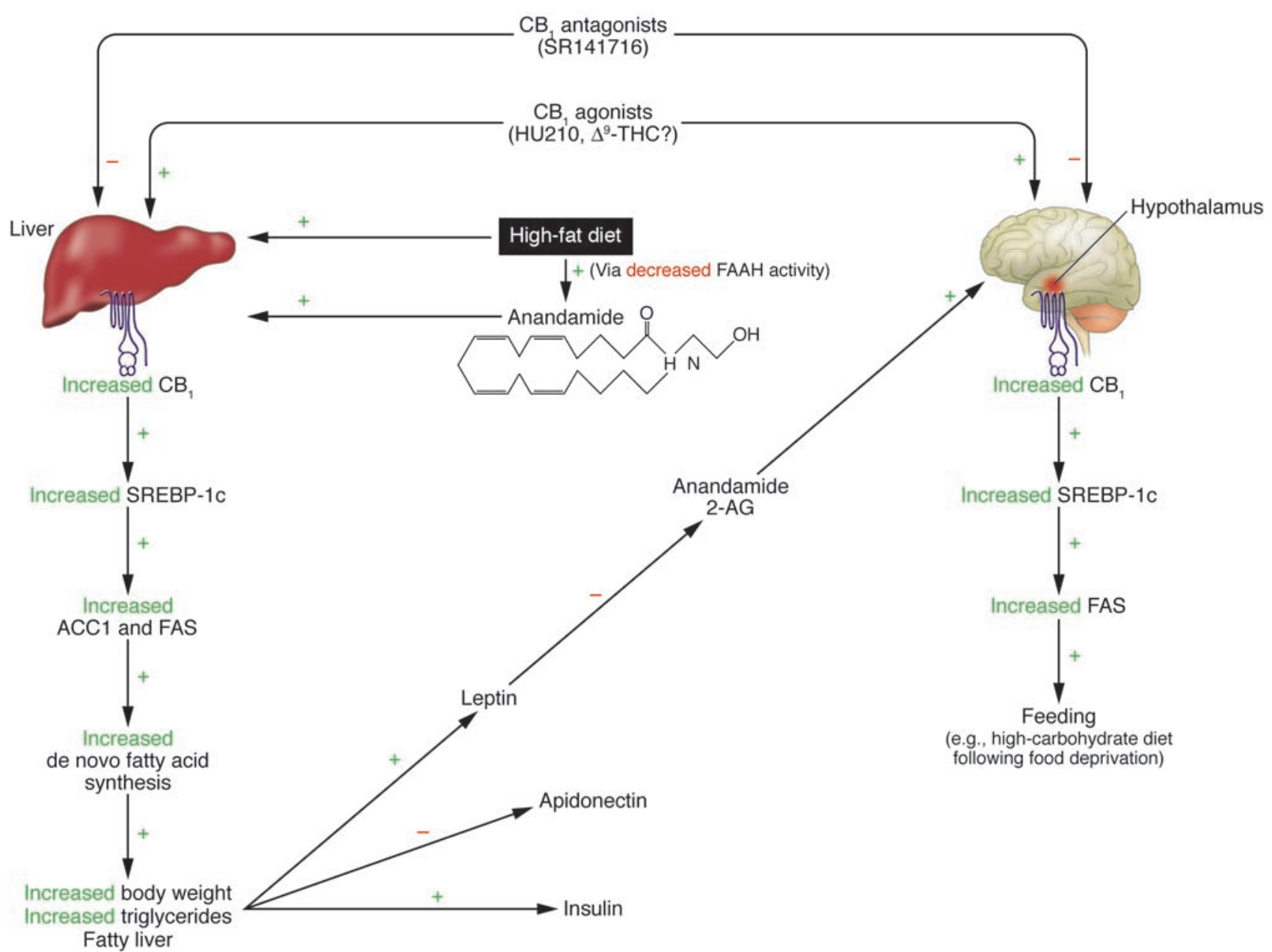

\section{Figure 1}

Proposed model for the dual role of the endocannabinoid-mediated pathway in regulation of peripheral metabolic and central appetitive processes in liver and hypothalamus. In liver (left), a high-fat diet leads to increased $\mathrm{CB}_{1}$ levels as well as increased levels of anandamide, the latter of which result from decreased activity of $\mathrm{FAAH}$, the primary enzyme responsible for this endocannabinoid's catabolism. $\mathrm{CB}_{1}$ stimulation increases expression of the transcription factor SREBP-1c and its associated enzymes, ACC1 and FAS. Stimulation of this pathway leads to a functional increase in the rate of de novo fatty acid synthesis in liver and the increased occurrence of fatty liver and obesity. Through either direct or indirect mechanisms, this pathway also regulates plasma levels of hormones associated with metabolism and feeding, including insulin, leptin, and adiponectin. These altered levels of hormones may negatively affect feeding behavior and metabolism. For example, exogenous administration of leptin leads to decreased levels of anandamide and 2-AG in the hypothalamus and consequently inhibits feeding behavior. Conversely, stimulation of $\mathrm{CB}_{1}$ in the hypothalamus (right) activates SREBP-1C and FAS, which leads to a hyperphagic response to a high-carbohydrate meal following a fast. Disruption of $\mathrm{CB}_{1}$ signaling through the use of $\mathrm{CB}_{1}{ }^{-1-}$ mice or SR141716 (Rimonabant) administration blocks both pathways, leading to a net effect of decreased fatty acid production and prevention of hyperphagia.

stimulates lipogenesis in primary adipocyte cell cultures (19). The presence of $\mathrm{CB}_{1} \mathrm{mRNA}$ in fat tissue from wild-type mice is consistent with a direct role of endocannabinoids in the regulation of lipogenesis.

\section{$\mathrm{CB}_{1}$ activation of SREBP-1c in liver and hypothalamus}

In this issue of the JCI, Osei-Hyiaman and colleagues identify the liver as a primary site responsible for endocannabinoid-mediated modulation of lipogenesis (23). Moreover, they present compelling evidence supporting a common molecu- lar pathway for peripheral lipogenic and central appetite regulation by an endocannabinoid signaling system, which involves the lipogenic transcription factor SREBP-1c (Figure 1) (24). Systemic administration of the potent cannabinoid receptor agonist HU210 led to significant increases in mRNA and protein levels of SREBP-1c, as well as those of its target enzymes, acetyl-CoA carboxylase-1 (ACC1) and fatty acid synthase (FAS), in liver. A functional consequence of the activation of these proteins was a 2 -fold increase in the rate of fatty acid synthesis in liver, an effect that was blocked by SR141716 administration and did not occur in $\mathrm{CB}_{1}^{-/-}$ mice. HU210 also increased SREBP-1c and FAS mRNA levels in the hypothalamus through a $\mathrm{CB}_{1}$-mediated mechanism. Although SR141716 given alone failed to affect basal hypothalamic mRNA levels of SREBP-1c and FAS in both mice that had food available ad libitum and those that were food-deprived, it blocked the increase of both of these transcripts in mice that were given access to a high-carbohydrate diet following 24 hours of food deprivation. Regardless, the observation that $\mathrm{CB}_{1}{ }^{+/+}$and $\mathrm{CB}_{1}^{-/-}$mice had a similar total caloric intake, independent of 
diet, supports the assertion that increased cannabinoid-mediated lipogenesis plays a more dominant role in diet-induced obesity than does hyperphagia. In addition to calorie intake and lipogenesis, other factors such as resting metabolic rate, total energy expenditure, and dietary-induced thermogenesis play a critical role in regulating body weight. Presently, the relationship between endocannabinoids and methods of energy expenditure is largely unknown, and the possibility that endocannabinoids may modulate these processes cannot be negated.

A particularly provocative implication of the work by Osei-Hyiaman et al. (23) is that endocannabinoid-mediated modulation of lipogenesis in the liver may contribute to the development of fatty liver and obesity. Wild-type mice maintained on a high-fat diet gained significant amounts of weight, mostly in the form of adipose tissue, and had altered levels of enzymes associated with metabolism, including insulin, leptin, and adiponectin. Additionally, these mice had an increased rate of hepatic fatty acid synthesis, showed increased levels of triglycerides, and developed fatty liver. In contrast, $\mathrm{CB}_{1}{ }^{-/-}$mice maintained on a high-fat diet did not exhibit increases in fatty acid synthesis, exhibited serum hormone and lipid profiles similar to wild-type mice fed regular chow, and did not develop fatty liver. Similarly, SR141716 administration significantly attenuated the elevation in hepatic fatty acid synthesis in wild-type mice fed a high-fat diet. These findings are corroborated in other reports $(25,26)$, in which chronic SR141716 treatment of mice maintained on high-fat diets was found to have similar beneficial effects with respect to serum lipids, leptin, and body weight as those observed in $\mathrm{CB}_{1}^{-/-}$mice.

Another important finding reported by Osei-Hyiaman et al. is that the endogenous cannabinoid system in the liver undergoes adaptive changes in response to diet (23). Wild-type mice maintained on a high-fat diet developed fatty liver associated with significant increases in hepatic levels of $\mathrm{CB}_{1}$ and anandamide. Hepatic 2-AG levels were unaffected by diet, which suggests that anandamide is the predominant endocannabinoid signaling molecule in this liver pathway. The elevated levels of hepatic anandamide appear to be due to a decrease in activity of fatty acid amide hydrolase (FAAH), the primary enzyme responsible for anandamide catabolism in vivo (27). Curiously, the level of FAAH protein in liver was unaffected by diet, suggesting that the highfat diet led to a decrease in anandamide deg- radation, possibly the result of the elevated levels of fatty acids and other endogenous factors competing for FAAH. In contrast, $\mathrm{N}$-acyltransferase, an enzyme regulating anandamide synthesis (28), was unaffected.

Collectively, Osei-Hyiaman and colleagues have demonstrated that stimulation of $\mathrm{CB}_{1}$ in liver with $\mathrm{HU} 210$ leads to an increased rate of hepatic fatty acid synthesis through a mechanism involving increases in SREBP-1c and its target enzymes, ACC1 and FAS (23). Similarly, they report intriguing evidence that anandamide activation of $\mathrm{CB}_{1}$ in liver is necessary for increased fatty acid synthesis following consumption of a high-fat diet. According to this model, consumption of a diet high in fats would lead to increases in fatty acid synthesis through the upregulation of endocannabinoid-mediated SREBP-1c in liver. Future work will need to address the issue of whether conditions that elevate hepatic anandamide levels are sufficient to stimulate SREBP-1c, ACC1, and FAS. Increasing the storage of fat could conceivably be an adaptive advantage in situations in which food is scarce, but could contribute to obesity, along with decreases in energy expenditure, when high-fat diets are consumed on a regular basis. A similar pathway involving endocannabinoid modulation of SREBP-1c in the hypothalamus may account for increased feeding behavior following periods of food deprivation. Both pathways are likely to contribute to the purported therapeutic efficacy of $\mathrm{CB}_{1}$ antagonists for the treatment of obesity and hyperlipidemia. Nonetheless, the devastating impact that the disruption of $\mathrm{CB}_{1}$ signaling has on feeding and growth in young mice $(19,29)$ necessitates that caution should be applied for the use of this approach in pregnant women and nursing mothers. Conversely, stimulation of hepatic and hypothalamic endocannabinoid pathways may account for the efficacy of $\Delta^{9}$-THC in the treatment of wasting syndromes associated with AIDS and cancer.

Address correspondence to: Aron H. Lichtman, Department of Pharmacology and Toxicology, Box 980613, Medical College of Virginia Campus, Virginia Commonwealth University, Richmond, Virginia 23298-0613, USA. Phone: (804) 828-8480; Fax: (804) 8282117; E-mail: alichtma@hsc.vcu.edu.

\footnotetext{
1. Abel, E.L. 1980. Marijuana: the first twelve thousand years. Plemum Press. New York, New York, USA. 289 pp.

2. Gaoni, Y., and Mechoulam, R. 1964. Isolation, structure, and partial synthesis of an active constituent of hashish. J. Am. Chem. Soc. 86:1646-1647.

3. Berry, E.M., and Mechoulam, R. 2002. Tetrahydrocannabinol and endocannabinoids in feeding and
}

appetite. Pharmacol. Ther. 95:185-190.

4. Beal, J.A., et al. 1997. Long-term efficacy and safety of dronabinol for acquired immunodeficiency syndrome-associated anorexia. J. Pain Symptom Manage. 14:7-14.

5. Matsuda, L.A., Bonner, T.I., and Lolait, S.J. 1993. Localization of cannabinoid receptor mRNA in rat brain. J. Comp. Neurol. 327:535-550.

6. Munro, S., Thomas, K.L., and Abu-Shaar, M. 1993. Molecular characterization of a peripheral receptor for cannabinoids. Nature. 365:61-64.

7. Devane, W.A., et al. 1992. Isolation and structure of a brain constituent that binds to the cannabinoid receptor. Science. 258:1946-1949.

8. Sugiura, T., et al. 1995. 2-Arachidonoyglycerol: A possible endogenous cannabinoid receptor ligand in brain. Biochem. Biophys. Res. Comm. 215:89-97.

9. Calignano, A., La Rana, G., Giuffrida, A., and Piomelli, D. 1998. Control of pain initiation by endogenous cannabinoids. Nature. 394:277-281.

10. Walker, J.M., Huang, S.M., Strangman, N.M., Tsou, K., and Sanudo-Pena, M.C. 1999. Pain modulation by release of the endogenous cannabinoid anandamide. Proc. Natl. Acad. Sci. U. S. A. 96:12198-12203.

11. Terranova, J.P., et al. 1996. Improvement of memory in rodents by the selective CB1 cannabinoid receptor antagonist, SR 141716. Psychopharmacol. 126:165-172.

12. Marsicano, G., et al. 2002. The endogenous cannabinoid system controls extinction of aversive memories. Nature. 418:530-534.

13. Ledent, C., et al. 1999. Unresponsiveness to cannabinoids and reduced addictive effects of opiates in CB1 receptor knockout mice. Science. 283:401-404.

14. Marsicano, G., et al. 2003. CB1 cannabinoid receptors and on-demand defense against excitotoxicity. Science. 302:84-88.

15. Di Marzo, V., et al. 2001. Leptin-regulated endocannabinoids are involved in maintaining food intake. Nature. 410:822-825.

16. Rinaldi-Carmona, M., et al. 1994. SR141716A, a potent and selective antagonist of the brain cannabinoid receptor. FEBS Lett. 350:240-244.

17. Zimmer, A., Zimmer, A.M., Hohmann, A.G., Herkenham, M., and Bonner, T.I. 1999. Increased mortality, hypoactivity, and hypoalgesia in cannabinoid CB1 receptor knockout mice. Proc. Natl. Acad. Sci. U. S. A. 96:5780-5785.

18. Arnone, M., et al. 1997. Selective inhibition of sucrose and ethanol intake by SR 141716, an antagonist of central cannabinoid (CB1) receptors. Psychopharmacol. 132:104-106.

19. Cota, D., et al. 2003. The endogenous cannabinoid system affects energy balance via central orexigenic drive and peripheral lipogenesis. J. Clin. Invest. 112:423-431. doi:10.1172/JCI200317725.

20. Ravinet Trillou, C., Delgorge, C., Menet, C., Arnone, M., and Soubrie, P. 2004. CB1 cannabinoid receptor knockout in mice leads to leanness, resistance to diet-induced obesity and enhanced leptin sensitivity. Int. J. Obes. Relat. Metab. Disord. 28:640-648.

21. Bensaid, M., et al. 2003. The cannabinoid CB1 receptor antagonist SR141716 increases Acrp30 mRNA expression in adipose tissue of obese $\mathrm{fa} / \mathrm{fa}$ rats and in cultured adipocyte cells. Mol. Pharmacol. 63:908-914.

22. Liu, Y.L., Connoley, I.P., Wilson, C.A., and Stock, M.J. 2005. Effects of the cannabinoid CB1 receptor antagonist SR141716 on oxygen consumption and soleus muscle glucose uptake in Lep(ob)/Lep(ob) mice. Int. J. Obes. Relat. Metab. Disord. 29:183-187.

23. Osei-Hyiaman, D., et al. 2005. Endocannabinoid activation at hepatic $\mathrm{CB}_{1}$ receptors stimulates fatty acid synthesis and contributes to diet-induced obesity. J. Clin. Invest. 115:1298-1305. doi:10.1172/ JCI200523057.

24. Hua, X., Wu, J., Goldstein, J.L., Brown, M.S., and Hobbs, H.H. 1995. Structure of the human gene encoding sterol regulatory element binding pro- 
tein-1 (SREBF1) and localization of SREBF1 and SREBF2 to chromosomes $17 \mathrm{p} 11.2$ and $22 \mathrm{q} 13$. Genomics. 25:667-673.

25. Ravinet Trillou, C., et al. 2003. Anti-obesity effect of SR141716, a CB1 receptor antagonist, in dietinduced obese mice. Am. J. Physiol. Regul. Integr. Comp. Physiol. 284:R345-R353.
26. Poirier, B., et al. 2005. The anti-obesity effect of rimonabant is associated with an improved serum lipid profile. Diabetes Obes. Metab. 7:65-72.

27. Cravatt, B.F., et al. 2001. Supersensitivity to anandamide and enhanced endogenous cannabinoid signaling in mice lacking fatty acid amide hydrolase. Proc. Natl. Acad. Sci. U. S. A. 98:9371-9376.
28. Hillard, C.J., and Campbell, W.B. 1997. Biochemistry and pharmacology of arachidonylethanolamide, a putative endogenous cannabinoid. J. Lipid Res. 38:2383-2398.

29. Fride, E., et al. 2001. Critical role of the endogenous cannabinoid system in mouse pup suckling and growth. Eur. J. Pharmacol. 419:207-214.

\title{
Chemokine-mediated angiogenesis: an essential link in the evolution of airway fibrosis?
}

\author{
Ivor S. Douglas ${ }^{1,2}$ and Mark R. Nicolls ${ }^{1}$ \\ 1Department of Medicine, Pulmonary Sciences and Critical Care Medicine and 2 Denver Health Medical Center, \\ University of Colorado Health Sciences Center, Denver, Colorado, USA.
}

\begin{abstract}
Angiogenesis may be an important factor in the development of fibrotic lung disease. Prior studies have strongly suggested a role for angiogenic vascular remodeling in pulmonary fibrosis, and emerging evidence indicates that new vessel formation is critical in airway fibrosis. Bronchiolitis obliterans syndrome is a fibrotic occlusion of distal airways that is largely responsible for the morbidity and mortality of patients after lung transplantation. In this issue, Belperio et al. demonstrate a role for CXC chemokine receptor 2 in the regulation of angiogenesis-mediated airway fibroproliferation (see the related article beginning on page 1150). By integrating an understanding of neovascularization into the study of events that occur between inflammation and fibrosis, it becomes increasingly possible to rationally design therapies that can halt conditions of maladaptive fibrosis.
\end{abstract}

Neovascularization is an important component of fibrotic responses (1). In this issue of the JCI, Belperio and colleagues extend this relationship to the development of chronic lung transplant rejection (2). Using bronchoalveolar lavage fluid from patients with pending or established bronchiolitis obliterans syndrome (BOS) and tracheal allograft tissue from a mouse model of obliterative airway disease, the authors make a convincing case for the central role of CXC chemokine receptor 2 (CXCR2) regulation of angiogenesis-mediated airway fibroproliferation.

\section{Airway inflammation and fibrosis in the evolution of BOS}

Chronic allograft rejection is the chief factor limiting long-term survival following lung transplantation. BOS is the pathological correlate of chronic rejection and primarily

Nonstandard abbreviations used: BOS, bronchiolitis obliterans syndrome; CXCL, CXC chemokine ligand; CXCR2, CXC chemokine receptor 2.

Conflict of interest: The authors have declared that no conflict of interest exists.

Citation for this article: J. Clin. Invest. 115:1133-1136 (2005). doi:10.1172/JCI200525193 affects the respiratory and terminal bronchioles, which culminates in a fibrotic occlusion of the distal airways (3). The cumulative incidence of $\mathrm{BOS}$ at 5 years after lung transplant is between $50 \%$ and $80 \%$, and 5 -year survival after BOS onset is only $30-50 \%$. The International Society of Heart and Lung Transplantation Registry has noted that the development of BOS within the first year after transplantation is the single most important factor influencing 5-year mortality among patients undergoing lung transplantation (3). As a fibrotic disease, BOS is poorly responsive to standard immunosuppression employed by transplant physicians. Similarly, pulmonary fibrosis, which affects the lung interstitium rather than the conducting airways, responds poorly to immunotherapy and has long been associated with pathologic angiogenesis (4).

It is a generally recognized phenomenon that inflammation is an initiating event that precedes the progression to fibrosis in several lung diseases, including BOS and idiopathic pulmonary fibrosis. While fibrosis may be a frequent sequel of an acute or subacute inflammatory event, it is also clear that inflammation does not always result in fibrosis. The long-term effect of interstitial or airway fibrosis is irreversible lung architectural remodeling. Key questions regarding the mechanisms of airway remodeling are: (a) What are the specific inflammatory initiators? and (b) What is the sequence of events that culminates in fibroproliferation? In lung transplantation, the answer to the first question most certainly involves the response to alloantigen triggering of innate and adaptive immune responses. The answer to the second question is probably less well understood but is perhaps of greater importance in the development of therapies that reach beyond immunosuppression. Lung transplant clinicians well appreciate that acute rejection treated early may respond excellently to immunosuppressive therapies but that late intervention is rarely successful. Unfortunately, it is not always possible to intervene early, and occasionally even apparently early intervention with highdose steroids or $\mathrm{T}$ cell-depleting strategies cannot halt the decline in lung function once fibroproliferation is initiated.

\section{The potential role of CXC chemokines in angiofibroproliferative BOS}

The study by Belperio and colleagues (2) firmly establishes that neovascularization is an important contributor to the process of fibroproliferation in airway fibrosis. The investigators present a cohesive and clearly argued interpretation of experimental data from human BOS patients and a well-characterized murine model of tracheal transplant rejection. Their findings make a convincing case for the central role of CXCR2-dependent Glu-LeuArg-positive $\left(\mathrm{ELR}^{+}\right)$chemokine regulation of angiogenesis-mediated BOS fibroproliferation. The study extends their previ- 